\title{
Norois
}

Environnement, aménagement, société

$208 \mid 2008 / 3$

Gestion du littoral, risques urbains, ours en Pyrénées

\section{La restauration de l'ours brun (Ursus arctos) dans les Pyrénées françaises : entre politique environnementale et crise-mutation du monde agricole}

The restoration of the Brown Bear (Ursus arctos) in the French Pyrenees: between environmental politics and crisis-change of the agrarian society

\section{Farid Benhammou et Marie Coquet}

\section{(2penEdition}

\section{Journals}

Édition électronique

URL : https://journals.openedition.org/norois/2224

DOI : $10.4000 /$ norois.2224

ISBN : 978-2-7535-1556-7

ISSN : 1760-8546

Éditeur

Presses universitaires de Rennes

Édition imprimée

Date de publication : 1 novembre 2008

Pagination : 75-90

ISBN : 978-2-7535-0788-3

ISSN : 0029-182X

Référence électronique

Farid Benhammou et Marie Coquet, «La restauration de l'ours brun (Ursus arctos) dans les Pyrénées françaises : entre politique environnementale et crise-mutation du monde agricole », Norois [En ligne], 208 | 2008/3, mis en ligne le 01 novembre 2010, consulté le 14 janvier 2022. URL : http:// journals.openedition.org/norois/2224 ; DOI : https://doi.org/10.4000/norois.2224 


\title{
LA RESTAURATION DE L'OURS BRUN (URSUS ARCTOS) \\ DANS LES PyrÉnées FRANÇAISES : ENTRE POLITIQUE ENVIRONNEMENTALE ET CRISE-MUTATION DU MONDE AGRICOLE
}

\author{
FARID BENHAMMOU \\ Équipe Recherche en gestion sur les territoires et l'environnement, \\ CIRED - ENGREF/Agro Paris Tech \\ 2 rue Saint-Paul - 45000 OrLÉANS \\ benhammou@engref.fr \\ Marie Coquet \\ ESO CARTA \\ (Université d'Angers), \\ 7 allée François-Mitterrand, BP 40455 - 49004 ANGERS \\ mariecoquet@free.fr
}

\begin{abstract}
RÉSUMÉ
La situation de l'ours brun dans les Pyrénées françaises laisse peu de personnes indifférentes, d'autant plus qu'elle occupe régulièrement la scène médiatique. La réintroduction de 2006 a fait oublier celle de 1996 et l'existence d'une population autochtone. L'opposition des points de vue nuit à la compréhension de la complexité des enjeux écologiques, territoriaux, politiques et culturels liés à ce plan de renforcement. La frange radicale hostile à l'ours a semé le trouble dans les esprits. Des maladresses des pouvoirs publics et surtout la crise-mutation des sociétés rurales ont contribué à lui donner du poids dans le débat. Pourtant, même localement, les choses sont partagées et les opinions favorables sont souvent apparues majoritaires. Le devenir du «Seigneur des montagnes » comme l'appelaient les Pyrénéens n'est pas assuré. Nous reviendrons sur la cohabitation ancienne de l'ours et des hommes dans les Pyrénées françaises. Les spécificités culturelles induites par cette coexistence sur ce territoire sont considérables. Les opposants à l'ours évoquent avec force l'importance de sauvegarder le " patrimoine » pyrénéen annoncé comme incompatible avec le retour de l'ours. Pourtant, il apparaît que ce " patrimoine» mal défini et les équilibres socio-économiques des zones rurales concernées étaient menacés avant cette politique environnementale qui fait office d'exutoire. L'objet de notre réflexion visera aussi à réfléchir à un avenir possible des sociétés rurales montagnardes, confrontées à un point de rupture de leur modèle, révélé par l'ours. Il est alors le symbole fort non seulement d'une restauration de la biodiversité, mais plus globalement de la manière dont les sociétés s'insèrent - ou pas - durablement dans un environnement fragile.
\end{abstract}

MoTS-CLÉ : ours - Pyrénées - sociétés rurales - montagne - patrimoine - environnement 


\section{ABSTRACT \\ The restoration of the Brown Bear (Ursus arctos) in the French Pyrenees: between environmental politics and crisis-change of the agrarian society}

Much people feel concerned with the situation of the Brown Bear in the French Pyrenees more especially as it occupies the media scene regularly. The reintroduction in 2006 made forget that of 1996 and the existence of an indigenous population. The opposition from the ideas harms the comprehension of the complexity of the ecological, territorial, political and cultural stakes related to this plan of reinforcement. The hostile radical fringe to Bear sowed the disorder in the spirits. This one has become more serious thanks to the public authorities awkwardness and especially thanks to the crisis-change of the rural companies. However, even locally, the things are confused and the favourable opinions often appeared majority. The future of the "Lord of the mountains" as Pyrenean called him, is not secure. We will reconsider Bear and men traditional cohabitation in the French Pyrenees later. The cultural identities induced by this coexistence on this territory are considerable. The opponents of Bear evoke strongly how it is important to safeguard Pyrenean heritage announced like incompatible with the Bear's come back. However, it appears that this badly definite "inheritance" and socio-economic balances of the rural areas concerned were threatened before this environmental policy which acts as discharge system. The meaning of our thought will also aim at thinking of a possible future of the mountain rural communities, confronted by a break with their model, revealed by Bear. Then It is the strong symbol not only for restoration of the biodiversity, but more generally in the way as the societies form part - or not-durably into a fragile environment.

\footnotetext{
KEY WORDS : bear - Pyrénées - mountain rural communities - heritage - environment
}

Le programme de renforcement de la population ursine était devenu indispensable pour éviter l'extinction totale des plantigrades dans les Pyrénées françaises. De fait, les mesures de sauvegarde prises par les gouvernements successifs depuis 1984 se sont avérées insuffisantes et inefficaces pour assurer la viabilité du noyau autochtone. L'arrivée de nouveaux plantigrades n'est pas passée inaperçue en 2006, alors que celle de 1996 a vite été oubliée. La virulence des conflits suscités par une petite frange radicale du monde agro-pastoral s'insère dans un dossier complexe. Pourtant les oppositions à l'ours s'inscrivent dans un champ plus vaste dont l'historique a déjà été réalisé par C. Dendaletche (1993), L. Mermet (2006 [1998]) et F. Benhammou (2001; 2005; 2007). A partir de 1994, les pouvoirs publics abandonnent la mission de conservation des ours autochtones du Haut-Béarn à un organisme de gestion dirigé par des élus locaux, initialement soutenu par l'Administration agricole (Mermet, 2001 ; Mermet, 2002). Malgré son discours, cette institution ${ }^{1}$ n'apparaît pas en mesure d'assurer la sauvegarde des derniers ours et tend même à bloquer la situation. À partir de 1996, les autorités décident alors d'encourager une réintroduction en Pyrénées centrales ${ }^{2}$, demandée par le regroupement de communes, $\mathrm{ADET}^{3}$-Pays de l'ours et l'association écologiste Artus. La population passe de 3 ours réintroduits à près d'une quinzaine en moins de 10 ans (fig. 1). Malgré des tensions périodiques, parfois fortes, l'état de conservation et l'acceptation sociale de l'espèce se sont améliorés dans cette période. Vu le faible nombre de femelles dans le noyau issu de la réintroduction et l'abattage de la dernière ourse pyrénéenne autochtone en Béarn (Pyrénées-Atlantiques) en 2004, un plan de renforcement voit le jour en

1. L'Institution patrimoniale du Haut-Béarn.

2. Les ours de cette région ont vraisemblablement disparu au début des années 1990.

3. Association pour le développement économique et touristique en Pyrénées centrales dans le Pays de l'ours. 
2005. Bien que demandé par l'opinion publique nationale et pyrénéenne, ce programme dynamise considérablement les opposants à l'ours. Malgré les tensions accompagnant les opérations de lâchers en 2006, ils se font là où il existe des élus favorables organisés, en Pyrénées centrales. La situation géopolitique du Béarn est davantage bloquée alors que la population ursine autochtone est condamnée faute de femelle (Benhammou, 2007).

L'objectif de notre propos est de revenir sur la géographie et l'histoire culturelle complexe des relations hommes-ours dans les Pyrénées. Longtemps délaissés par la géographie, les animaux, a fortiori la grande faune, se révèlent d'extraordinaires révélateurs et analyseurs de spécificités, d'évolutions socio-territoriales, politiques et écologiques (Blanc et Cohen, 2001, Benhammou, 2006; Benhammou, 2007; Pihet, 2006). À l'aune de cela, nous aborderons la désapprobation des militants anti-ours du monde agro-pastoral et politique des Pyrénées centrales. Ils jugent ce programme de renforcement inutile, onéreux voire dangereux pour l'avenir de l'activité agricole en montagne. Pour eux, les priorités sont mal distribuées. Pourtant, les partisans, dont certains issus du pastoralisme, estiment que l'arrivée de nouveaux ours est une nécessité voire une aubaine pour l'activité économique montagnarde. En découle une situation conflictuelle et explosive dans un territoire déjà éprouvé par de nombreuses difficultés. Pourquoi une telle cristallisation des conflits dans un territoire où la cohabitation avec l'ours est millénaire? La fin d'une crise-mutation du monde agricole et rurale nous semble, entre autre, une bonne piste de réflexion. Les opportunités de recherche sont considérables, car de nombreux enseignements sur les failles, les résistances et les perspectives des politiques d'environnement en général peuvent être tirés. Par ailleurs, il apparaît nécessaire de réfléchir à l'avenir des ours et de l'activité pastorale en Pyrénées françaises, à l'heure où le point de rupture du modèle actuel de développement de ces territoires semble sur le point d'opérer.

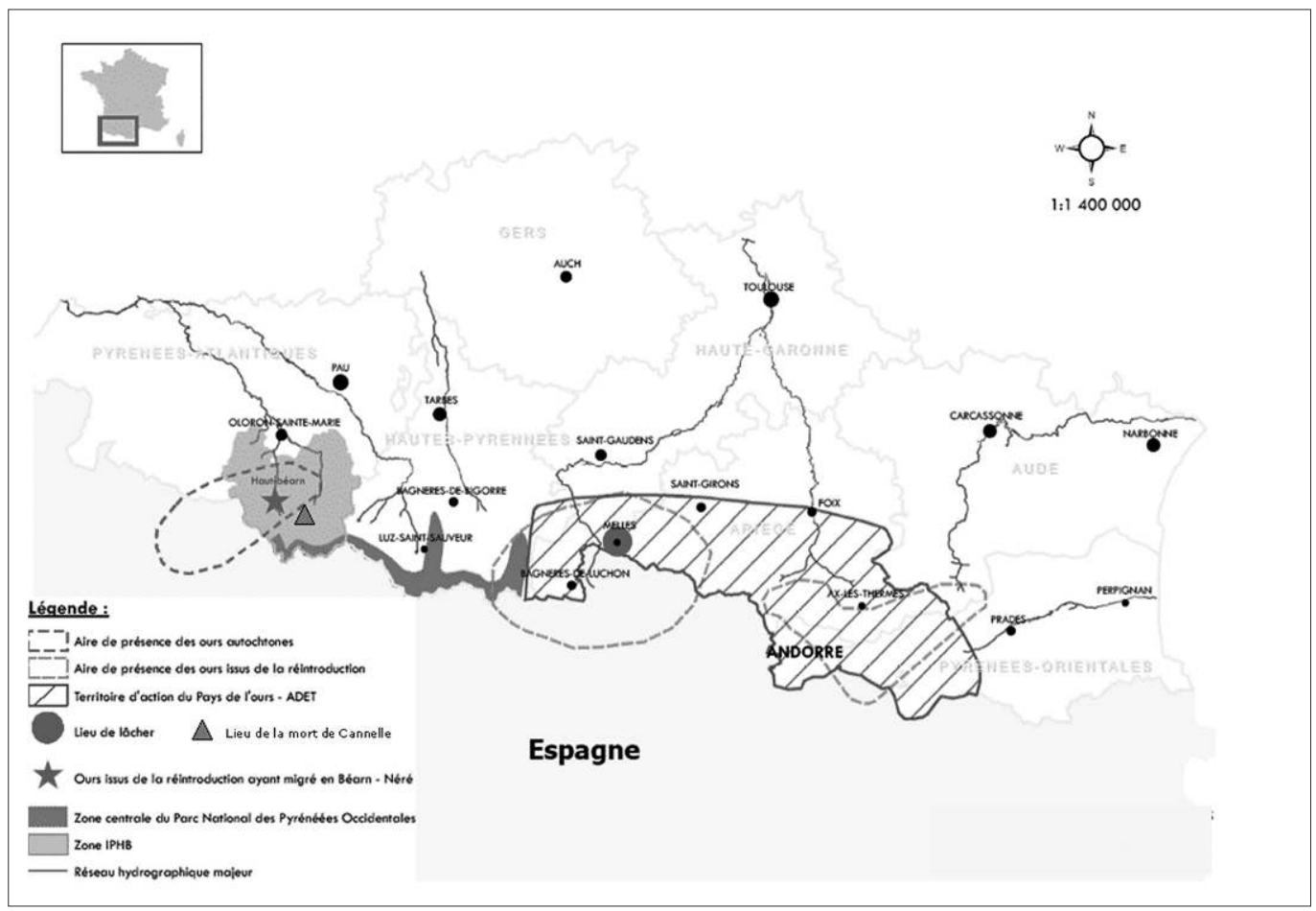

Figure 1 : Où sont les ours en 2005 (avant la $2^{\mathrm{e}}$ réintroduction de 2006)?

Location of the Bears in the Pyrenees before the $2^{\text {nd }}$ reintroduction in 2006 


\section{Une occupation du plantigrade ancienne mais menacée dans le massif pyrénéen}

\section{Le déclin territorial de L’ours en France et dans les Pyrénées}

Quelques rappels paraissent indispensables. La légitimité de l'occupation géographique de l'ours est souvent contestée. Pourtant, l'ours brun était, à l'origine, présent dans la quasi-totalité de l'Europe, et ce depuis près de 250000 ans (Camarra, 2005). En France, au néolithique ancien (-6000 à 4000 avant notre ère), il occupe la totalité du territoire métropolitain et des indices archéologiques montrent des traces de cultes à l'ours, notamment dans les Pyrénées. Le plantigrade, ayant une prédilection pour la forêt, n'est donc pas exclusivement un animal de montagne, même s'il s'y est bien adapté. Il y a été contraint par la pression humaine sur les milieux. Dès la période romaine, le déclin de l'ours est largement amorcé. Au fil des siècles, son aire de vie ne cesse de régresser. Ainsi, dès le XVIII ${ }^{\mathrm{e}}$ siècle, l'ours n'a pu subsister que dans des espaces géographiques restreints : Pyrénées, sud du Massif central et espaces forestiers montagnards des Alpes à la Franche-Comté. Ce phénomène est majoritairement dû aux battues, à la chasse et à la destruction de son habitat. En 1850, l'ours disparaît de Franche-Comté. Dès la fin du XIX ${ }^{e}$, son implantation dans le massif alpin est gravement menacée. Le dernier ours y est observé en 1937.

Dans les Pyrénées, au début du XXe siècle, la population ursine occupe encore la totalité du massif. Dès les années 1950, sa répartition se scinde (fig. 2).
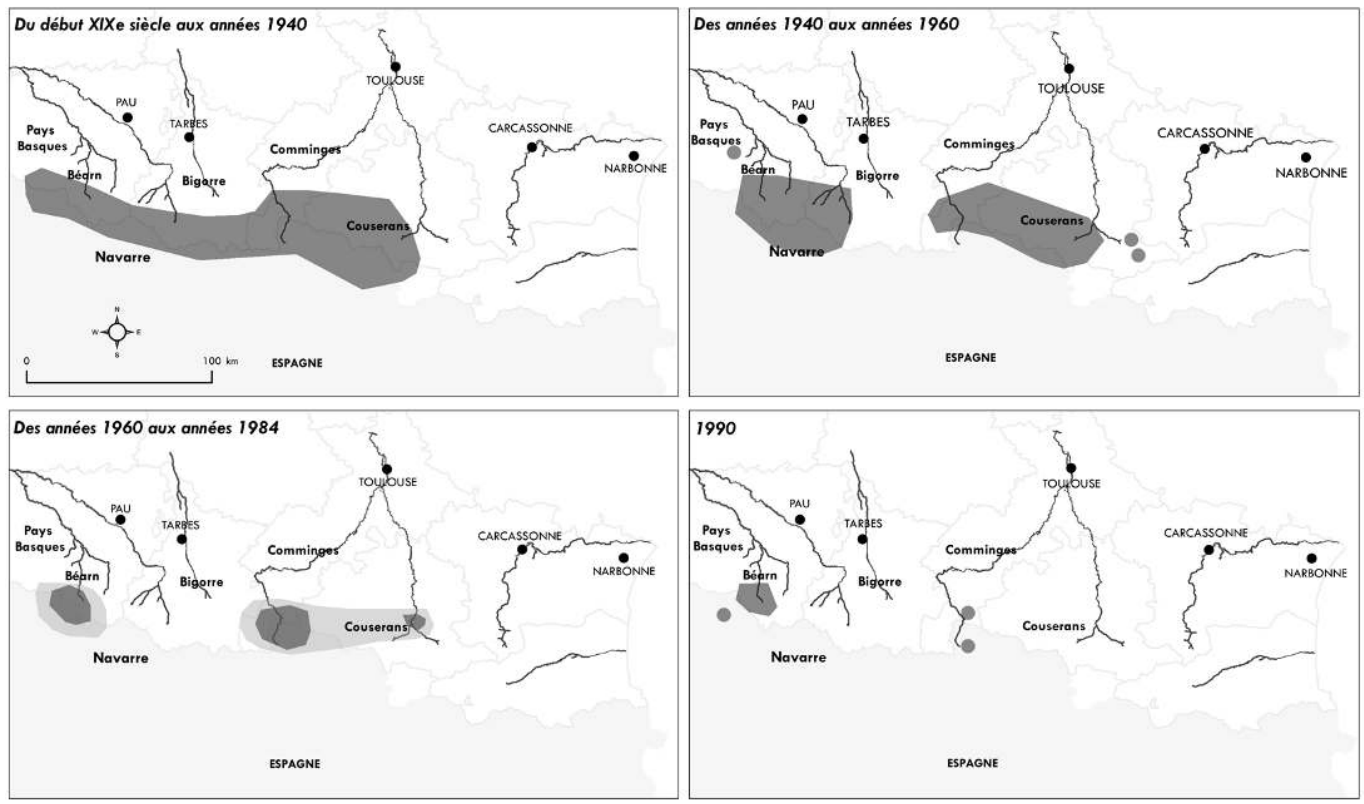

Aire de réaparition des ours

Zone de présence occasionnelle

Animaux erratiques

Figure 2 : La régression de la population d'ours dans les Pyrénées The decline of Bear population in the Pyrenees

Trois noyaux géographiques se distinguent peu à peu : Pyrénées orientales, Pyrénées centrales et Pyrénées occidentales avec le Béarn. Cette dernière zone devient l'ultime bastion des ours pyrénéens où ils ne sont plus que cinq ou six en 1995. Cette population a été marquée par une 
mortalité élevée - souvent anthropique - des femelles reproductrices (Etchelecou, 1995). En 2004, la dernière femelle de souche pyrénéenne est abattue par un chasseur. L'extinction de la branche autochtone devient inéluctable si rien n'est fait. Malgré cela, les opposants se disent réfractaires à la politique de renforcement, mais pas à la présence de l'ours dans le massif. Les deux vont néanmoins de pair : si aucune intervention extérieure n'a lieu, l'ours brun n'a aucune chance de survivre dans les Pyrénées.

En règle générale au plan mondial, le même constat peut-être établi : l'aire d'occupation des ours s'est restreinte de manière significative. L'ours brun ne peut être considéré comme une espèce en extension en Europe, sauf exception comme la Slovénie.

\section{L’ours : UNE PLACE PARTICULIÈRE DANS LE PATRIMOINE PYRÉNÉEN}

L'ours n'a jamais cessé d'occuper le massif pyrénéen, même si sa population, depuis quelques années ne s'est maintenue qu'à l'état de relique. Néanmoins, cette occupation ancienne du massif par le plantigrade a généré une spécificité culturelle.

L'ours y a été largement mythifié. Pendant longtemps, il fut considéré par les Pyrénéens comme un homme déchu, sauvage, mais proche d'eux. Les raisons de cette perception sont à chercher dans son apparence physique. Il se dresse régulièrement en position verticale, à la manière d'un être humain. Les empreintes de l'ours rappellent étrangement celles de pieds griffus. Les habitants des vallées pyrénéennes avaient d'ailleurs coutume de l'appeler le « pé descaou », le va-nupieds.

Son régime alimentaire, comme celui des humains, est omnivore. L'ourse allaite ses petits, souvent assise, comme une femme pourrait le faire. Ces analogies ne sont pas dénuées d'intérêt. Les réactions d'animosité à l'ours, souvent expliquées trop rapidement par une haine ancestrale, ne trouvent pas leur racine dans le patrimoine culturel pyrénéen. Les anciens avaient l'habitude de parler de lui avec une certaine déférence, faite de respect et de crainte. Il était habituel de le nommer avec des sobriquets tels que "maître ", «seigneur» «lou moussu » ou «le type ». Ces surnoms permettaient d'éviter, croyait-on, qu'il comprenne les conversations dont il était l'objet. Il était aussi commun de lui donner un prénom humain tels que Gaspard, Martin, Dominique... Cette pratique perdure aujourd'hui. L'ours fut donc longtemps considéré par les Pyrénéens comme ce parent éloigné, cet homme rustre, bourru, resté sauvage. Ce proverbe basque est d'ailleurs tout à fait significatif : "Ne tue pas l'ours, c'est ton père. »

Différentes légendes, comme celle de Jean de l'ours, peuvent être évoquées pour illustrer ce lien particulier entre l'homme et le plantigrade. O. de Marliave (2000), S. Bobbé (1998) en présentent plusieurs. Ainsi, dans les légendes pyrénéennes, l'ours est, soit le descendant, soit l'ascendant de l'homme. Les fêtes de l'ours au printemps émaillent le folklore pyrénéen de la Catalogne au

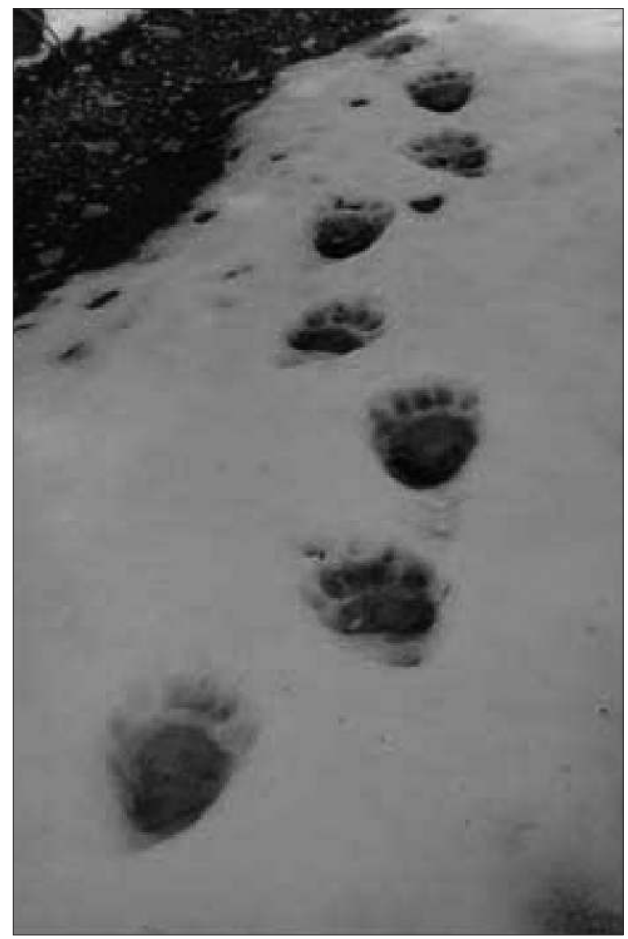

Photo 1 : Des empreintes d'ours proches de celle de l'homme (Source : [http://www.spi-ind.com/Our_ Forests/Images])

print
Bear footprints close to Human foots- 
Pays Basque. L'influence de l'animal est facilement visible dans les lieux. De nombreux toponymes (vallée d'Ossau, Osse-en-Aspe) et l'architecture locale en témoignent : sculptures de tête ou de silhouettes d'ours sur certaines façades, sur du mobilier urbain...

Si la cohabitation est ancienne et le folklore riche, cela ne veut pas forcément dire connaissance intime de l'espèce. En outre, ce savoir empirique sur l'espèce devait être meilleur par le passé, notamment quand il existait des chasseurs spécialisés quasi-professionnels (Mermet et Benhammou, 2005). Plusieurs idées sans fondement circulaient, comme le fait qu'un ours très prédateur est forcément étranger (ceci avant même la réintroduction). Ce cliché a été réactivé avec les lâchers d'ours slovènes. Or, des ours plus prédateurs que les autres ont toujours existé. L'origine n'est alors pas déterminante, mais plutôt l'absence de gardiennage, l'opportunisme et la « personnalité » des spécimens ou encore le faible degré de tolérance des éleveurs nouvellement concernés.

Par ailleurs, A. Reynes (2005) rappelle que « la guerre de l'ours » n'a historiquement pas eu lieu. À savoir que les Pyrénéens n’ont jamais cherché à exterminer le plantigrade, contrairement au loup par exemple. Ils avaient conscience qu'il existait des individus inoffensifs pour le bétail et ils ne détruisaient l'espèce que ponctuellement. Ce n'est qu'une fois atteint un seuil critique, fatidique pour une démographie peu dynamique, que sa perpétuation s’est avérée problématique.

\section{UNE DUALITÉ HISTORIQUE DES RAPPORTS OURS/SOCIÉTÉS RURALES : ENTRE FASCINATION ET REJET}

Les rapports entre l'éleveur-berger ${ }^{4}$ et l'ours sont complexes. Il est directement confronté à la présence de l'ours en raison de ses activités. Au travers de ses rencontres, de cette cohabitation est né un particularisme culturel propre aux Pyrénées. Réfléchir à l'ambiguïté de ces rapports paraît intéressant pour éclairer différemment les crispations actuelles. Le livre d'E. Lamazou, L'Ours et les brebis (1988), est instructif à cet égard. Ce berger transhumant vivant dans la vallée d'Aspe, dans le Béarn, dans le village d'Aydius, a exercé sa profession de 1913 à 1969. Il relate la fascination que l'animal exerçait sur lui lorsqu’il était enfant. "À vrai dire, j'avais une folle envie de le rencontrer, en chair et en os » (ibid.). L'occasion lui en est donnée alors qu'il garde le troupeau avec son grand-père. Ce dernier laisse l'animal s'approcher afin que son petit-fils puisse l'observer de plus près. Les hommes étaient donc habitués à cette cohabitation, à une époque où les ours étaient beaucoup plus nombreux dans le massif. Ils ne craignaient pas réellement pour leur sécurité même s'ils redoutaient l'incursion de l'animal sur les estives, pour leur cheptel. Cependant, toute appréhension n'était pas à exclure face à un animal impressionnant. À l'heure actuelle, les opposants à l'ours aimeraient lui faire endosser le rôle d'un animal dangereux. Pourtant, les Béarnais ayant toujours cohabité avec l'ours, E. Lamazou n'exprime pas de peur mais un mélange de sentiments, bien compréhensible. Lorsque le grand-père a estimé que l'ours s'était suffisamment approché de son troupeau, il se contenta de crier «Ahoü », utilisé de manière courante par les bergers pour faire fuir les prédateurs. Comme l'explique l'auteur, l'animal s'est enfui car il craint les contacts avec l'homme. Il évoque d'autres situations où l'animal est côtoyé, dont une fois une journée entière, sans qu'il ne représente la moindre menace pour lui, sa famille ou même le bétail. Néanmoins, il explique les désagréments que cette cohabitation a pu apporter au troupeau : "Que messieurs les écologistes ne soient pas troublés ou indignés par le fait que nous ne l'aimons guère. [...] Comment accepterions-nous, la joie au cœur, sous prétexte que l'espèce est menacée, que les ours viennent en [sous-entendu le troupeau] faire leur repas de fête? » (ibid.). Là encore, l'ambivalence des liens s'exprime pleinement, car il ajoute plus loin : « Il semblerait, d'après ce

4. En théorie, l'éleveur n'est que le propriétaire des bêtes et le berger, celui qui les garde. Ces personnages sont le plus souvent distincts, mais les éleveurs se font volontiers passer pour berger en raison du capital de sympathie bucolique attribué à la profession. Dans le Béarn, la persistance de bergers et/ou éleveurs fromagers, vivant longtemps sur l'estive, conduit à un contact prolongé avec les bêtes, à traire et à surveiller, avec le territoire et... les ours. La situation est différente en Pyrénées centrales où l'élevage extensif pour la viande domine (voir Benhammou, 2007). 
que disent les spécialistes qu'il ne reste plus assez d'ours pour assurer la reproduction saine de l'espèce. Même si j'ai versé comme tous les bergers un lourd tribut au seigneur de nos montagnes, je suis le premier à le regretter, car il en faisait intégralement partie, et sans lui, elle ne sera plus jamais tout à fait la même» (ibid.). Ce propos rejoint celui de J. Paroix (1999), berger en vallée d'Ossau ou encore de J. Labarrère, berger en vallée d'Aspe qui disait en 1998: «Moi, je n'ai pas honte de le dire, je regrette qu'il n'y ait plus d'ours. On ne dirait pas, mais c'était un compagnon du berger. On ne pouvait pas les séparer. Je crois qu'on va disparaître ensemble, ours et bergers » (Benhammou, 2001). L’ours est bel bien intégré au milieu montagnard pyrénéen au sens naturel mais aussi culturel.

Les similitudes avec l'homme ont complexifié les rapports avec l'animal. Tous deux positionnés en haut de la chaîne trophique, ils entrent en concurrence dans un espace géographique partagé. Certainement, la compétition perdure entre l'homme et le prédateur. Actuellement, les oppositions les plus fortes à l'ours se trouvent dans des zones localisées des Pyrénées centrales (Ariège, Pays Toy dans les Hautes-Pyrénées) ou auprès d'acteurs agro-pastoraux béarnais en rupture avec le pastoralisme traditionnel qui cohabite de longue date avec Ursus arctos. Car, au plan historique et culturel, on ne peut affirmer que l'ours n'était pas assimilé à la montagne et à ses habitants. Il était tout à la fois toléré, détesté et apprécié (Charlier, 1988). Ce sont ces rapports qui confèrent aux Pyrénées leur spécificité et leur authenticité tellement revendiquées par les locaux. C'est donc une société agro-pastorale en perte de repère qui voit certains de ses membres réagir de manière excessive au retour d'une espèce emblématique de la région. Ce constat est à mettre en lien avec la surreprésentation parmi les leaders anti-ours des néo-ruraux éleveurs ou des éleveurs d'origine locale ayant abandonné les pratiques de gardiennage traditionnel (Bellefon [de] et Benhammou, 2004 ; Benhammou, 2007).

\section{Sociétés rurales montagnardes et ours brun : quel avenir?}

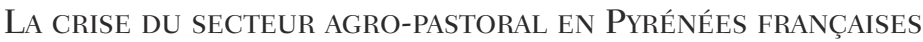

Le modèle agro-pastoral pyrénéen actuel se trouve confronté à de multiples difficultés. La situation n'est pas identique dans tout le massif. En Pyrénées-Atlantiques, la production ovine est davantage orientée vers le lait. En revanche, dans les départements des Hautes-Pyrénées, HauteGaronne et Ariège, l'élevage des brebis est consacré, dans sa plus grande partie, à la production d'agneau pour la viande. Ainsi, à l'ouest du massif, en Béarn notamment, la traite a longtemps induit la présence de bergers durant la période d'estive. Plus à l'est, la diminution du nombre de prédateurs a peu à peu conduit à l'abandon du gardiennage. Cette situation s'étend peu à peu au Béarn avec la disparition de l'ours. Aujourd'hui, le monde pastoral se trouve dans une situation difficile. La moyenne d'âge des actifs agricoles progresse. Les effectifs dans les exploitations ne cessent de décroître. Pourtant, le nombre de têtes par troupeau augmente. Le modèle est devenu extensif et intensif. Il est aujourd'hui difficile de vivre avec moins de 350 brebis et les revenus sont peu élevés. Les aides distribuées par la PAC ont surtout été attribuées selon des critères quantitatifs plus que qualitatifs. Les agneaux sont achetés à un prix dérisoire que viennent difficilement compenser les subventions agricoles. Mais sans elle, le travail n'est pas payé à sa juste valeur. Le prix de vente est resté inchangé depuis les années 1980 alors que le coût de la vie, lui, n’a cessé d'augmenter. D. Cheuzeville, éleveur à Arbas, explique que seules les grosses exploitations peuvent perdurer (Coquet, 2007) Actuellement, ce secteur agricole est celui qui dispose des plus faibles revenus de la profession. En 2006, alors qu'en moyenne le revenu des agriculteurs a progressé de $15 \%$, celui des éleveurs ovins a diminué de $2 \%$ (Bailly et Fortassin, 2008). Une première inadéquation peut être relevée. Le peu de cohérence des mesures prises dans le cadre de la transition de la nouvelle PAC en 2006 ulcère déjà le monde agricole. Malgré les discours ambiants, les aides au pastoralisme sont pénalisées et les nouvelles conditions d'obtention sont très complexes. Les renforcements ursins arrivent dans un secteur déjà éprouvé et la coïncidence avec cette phase 
de transition aiguë permet d'éclairer la violence de certaines réactions. Les objectifs des pouvoirs publics ne sont pas correctement perçus car ils ne s'articulent pas de façon logique. Dans ce contexte difficile, des leaders professionnels agricoles et des élus tendent à utiliser le mal-être ambiant contre les politiques environnementales (Natura 2000, limitation d'usage de ressources, protection de certaines espèces). Elles sont présentées comme sans fondements, parachutées, alors que ces mêmes leaders agricoles et politiques n'ont pas été en mesure de lutter outre mesure contre la concentration agricole et la déprise rurale. Les programmes de restauration des milieux et des espèces en danger apparaissent alors comme des exutoires faciles dans la mesure où les acteurs environnementaux, présentés comme puissants, sont en général faibles. Même s'il représente une gêne supplémentaire à ne pas nier, l'ours semble avoir bon dos. Il permet d'éviter que les problèmes de fond soient réellement abordés.

La politique concernant la sauvegarde de l'ours a été discontinue et marquée par des choix opposés selon les régions du massif et les pressions agro-politiques locales. Quoi qu'il en soit, le système agro-pastoral actuel arrive à son point de rupture : avec ou sans l'ours, il demeure impossible qu'il puisse perdurer en l'état. Par ailleurs, la crise qui le touche était amorcée bien avant l'arrivée de nouveaux plantigrades dans le massif. L'ours n'est qu'un révélateur de cette crise et qu'un catalyseur puissant de mécontentement.

\section{UN PROGRAMME SOLLICITÉ LOCALEMENT, VOLONTARISTE MAIS CONTROVERSÉ}

Les réactions d'hostilité au programme de renforcement, très médiatisées en 2006 et 2007, font souvent fi de l'historique du dossier. Malgré le déclin avéré de l'ours, la France n'interdit sa chasse qu'en 1962. Dès cette période, des naturalistes demandent aux autorités de prendre de vraies mesures de protection pour éviter la destruction illégale de spécimens. Il faut attendre 1982 pour que l'espèce soit effectivement protégée. Entre-temps, plusieurs femelles ont été tuées (Etchelecou, 1995) et le Parc national des Pyrénées, créé en 1967, ne peut pas protéger les derniers ours dans la mesure où ses limites ne contiennent pas les espaces vitaux fréquentés par les ours. En 1990, quand des mesures de protection stricte sont prises pour ces espaces (réserves dites «Lalonde»), des élus locaux, des chasseurs et des représentants agricoles organisent une forte protestation, surtout en Béarn. Pourtant, peu de gens savent que des fonctionnaires du ministère de l'Agriculture attisent en sous-main les tensions et aident ces acteurs locaux à s'organiser pour pouvoir lancer de grands projets d'aménagement de la montagne (un réseau de pistes sylvo-pastorales permettant notamment l'exploitation forestière). L'objectif est d'affaiblir la nouvelle emprise territoriale des services de l'Environnement nuisant à l'imposition des modèles standards de développement (Mermet, 2008 [1996]; Benhammou, 2005). L'Institution patrimoniale du Haut-Béarn incarne cette alliance entre intérêts agricoles, élus locaux et certaines franges de l'État, au détriment des intérêts environnementaux. Malgré les discours environnementaux de façade de cette institution, la situation est alors bloquée en Béarn pour une protection efficace de l'animal.

En 1987, lors d'un colloque à Seix (Ariège), les futurs fondateurs de l'association pro-ours Artus lancent l'idée de réintroduire des spécimens pour sauver l'ours. Dans la volonté de lier cela à un projet de développement des Pyrénées centrales, l'ADET (Association pour le Développement Économique et Touristique qui devient, plus tard, Adet-Pays de l'Ours) est créée par quatre communes de Haute-Garonne en 1991. D'autres la rejoignent ensuite ainsi que divers acteurs du territoire. L'initiative a donc d'abord été insufflée à l'échelle locale avant d'être soutenue par les services du ministère de l'Environnement. Les premiers lâchers n'interviennent qu'en 1996 en Pyrénées centrales, à Melles (Haute-Garonne). Il ne reste alors plus que cinq ours dans le massif, beaucoup plus à l'ouest, la survie du noyau autochtone n'étant toujours pas assurée.

En outre, les fédérations des chasseurs des Pyrénées centrales soutiennent l'opération car ils sont associés à l'équipe de suivi de l'espèce. Ils ont obtenu de ne subir aucune contrainte quant à leur activité. Ces éléments rappellent que la réintroduction de 1996, puis le renforcement de 2006 ont été, au départ, soutenus localement. Après la mort de Cannelle et à la suite du constat 
LA RESTAURATION DE L'OURS BRUN DANS LES PyRÉNÉES FRANÇAISES

d'échec de la préservation de la population ursine, des associations pyrénéennes, dont ADET-Pays de l'ours, et non pyrénéennes demandent de nouveaux lâchers d'ours.

\section{Politique DE RENFORCEMENT :}

\section{CONTRAINTES ET QUESTIONNEMENTS SUR UNE POLITIQUE ENVIRONNEMENTALE}

La première vraie opposition forte au plan de réintroduction en Pyrénées centrales ne s'exprime qu'en août 1999, soit près de 3 ans après les premiers lâchers. Cette saison d'estive a été marquée par un été sans soleil, de graves intempéries (neiges, grêles, brusques redoux, brouillard prolongé) et de nombreuses bêtes ont été perdues toutes causes confondues. Le terrain était donc bien préparé à une susceptibilité particulière aux dégâts d'ours et à l'amorce d'une mobilisation plus vaste. Dans la foulée, le député ariégeois A. Bonrepaux propose un amendement à la loi chasse en 2000 demandant le retrait des ours réintroduits. Relayée par des éleveurs proche de lui politiquement, une impulsion anti-ours est lancée, en rejoint et en fédère d'autres à travers le massif (Benhammou, 2001; Benhammou et Mermet, 2003). Même si le mouvement général s'essouffle à l'échelle des Pyrénées à partir de 2002, des foyers d'opposition radicaux, localisés, perdurent ou sont en dormance. C'est sur cette base que la mobilisation anti-ours reprend de l'ampleur à partir de 2005-2006.

Suit alors un conflit qui prend souvent la forme de joutes argumentaires. Les opposants à l'ours avancent que ces «nouveaux » ours seraient une menace pour le massif car ils participeraient à l'abandon des pâturages d'estive. Les éleveurs viendraient à les délaisser en raison des conditions de travail difficile, le retour du prédateur serait l'élément de trop. Le résultat induirait la reconquête du massif forestier à des altitudes toujours plus basses sur les versants. Les paysages remarquables liés à la tradition pastorale ancestrale (pelouse et prairies entretenues par le bétail) seraient menacés par l'arrivée des ours slovènes. Pourtant, les usages pastoraux entretenant les paysages avaient déjà largement tendance à décroître avant cela, en raison de la baisse du nombre d'exploitations, l'abandon de la transhumance et d'un grand nombre de prairies de fauche (Benhammou, 2003). La reconquête du couvert forestier était déjà une réalité. D’ailleurs, elle touche l'ensemble des milieux ruraux français, reflet des difficultés et de la déprise agricole, a fortiori en montagne. Ensuite, vient inévitablement la question du choix des animaux à réintroduire. L'ours slovène serait moins montagnard que son cousin pyrénéen, plus familier de l'homme et différent d'un point de vue génétique. Les études conduites ont montré que l'ours slovène était adapté à ce programme, pour diverses raisons : similitudes de milieu d'origine, de l'espèce Ursus arctos, même souche génétique principale... (Quenette, 2000). E. Lamazou montre bien que les ours s'approchaient parfois relativement près des hommes, qu'ils se sont toujours rendus à l'orée de la forêt. La reconquête du couvert forestier induit donc inévitablement que les ours descendent plus bas en altitude. Mais le déroulement des lâchers de 2006 (fig. 3) pose assurément question.

Après la surmédiatisation du début de l'opération qui a dynamisé et radicalisé la mobilisation des éleveurs anti-ours, les autorités ont opté pour des lâchers dans une discrétion faisant songer à de la clandestinité. L'impression de passage en force dans un territoire déjà à cran a été renforcée. La viabilité à long terme semble compromise, or selon l'Union internationale pour la conservation de la nature (UICN), pour qu'une réintroduction réussisse, il faut que l'acceptation sociale soit forte pour éviter une nouvelle destruction de l'espèce. Cette mobilisation agro-politique en viendrait presque à faire oublier la demande pyrénéenne de sauvetage de l'ours exprimée à travers des collectifs associatifs et plusieurs sondages, tous majoritairement favorables à l'opération, y compris en zone de montagne (Benhammou, 2007). Mais il n'en demeure pas moins que le programme n'a pas tous les atouts de son côté, car de nombreuses incohérences demeurent. À titre d'exemple, les chasseurs, avec lesquels de nombreux accords ont été passés afin de s'assurer leur soutien, continuent de bénéficier de l'autorisation de pratiquer leur activité sur des territoires où l'on sait qu'il y a potentiellement des femelles accompagnées de petits avec tous les risques que cela comporte. 


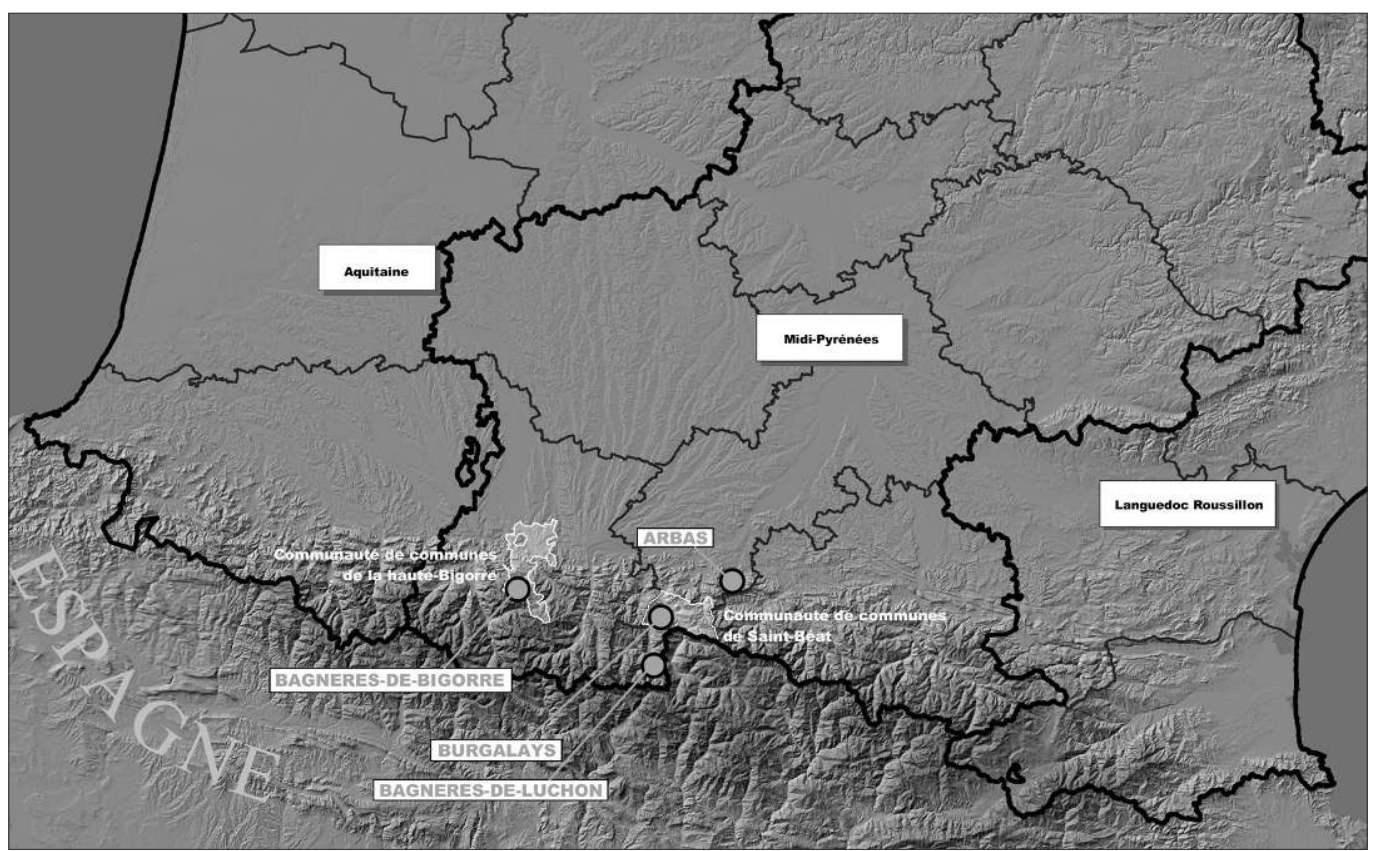

Figure 3 : Site de lâchers des ours en 2006 (Source : ADET-Pays de l'ours, ETO, 2006) Site of Bears release in 2006

Par ailleurs, d'un point de vue éthique et concernant le respect même de la vie animale la façon dont s'opère le programme est discutable. Les ours sont catapultés dans un territoire hostile en partie, et parfois dans la précipitation. Ce fut le cas pour Palouma dont le lâcher initialement prévu à Arbas fut interrompu par quelques opposants, en avril 2006 et donc opéré de nuit dans la commune de Burgalays. Le président du conseil scientifique du Parc national des Pyrénées, A. Etchelecou, a témoigné de son hostilité aux lâchers des ours de Slovénie. Démographe et géographe, militant de la première heure favorable à la cause de l'ours des Pyrénées, il estime que si les Français n'ont pas été capables de sauver la population ursine autochtone, ce n'est pas la peine de remettre des ours qui seraient sûrement mieux dans leur pays d'origine. Des éléments vont dans ce sens en apparence. Par exemple, l'ourse Palouma est morte en 2006 dans une chute accidentelle en montagne et Franska a été renversée par une voiture en août 2007 dans les Hautes-Pyrénées. Elle avait été perturbée tout l'été par les opposants à l'ours, ce qui l'a peut-être désorientée. Cependant, il arrive que des animaux meurent de chute en montagne et partout où les ours et les hommes cohabitent, les plantigrades traversent les routes et s'exposent aux voitures ${ }^{5}$. Par ailleurs, alors que les ours sont chassés en Slovénie, ils ont plus de risque de s'y faire tuer que dans les Pyrénées.

L'ours a écologiquement, historiquement et culturellement une place dans les Pyrénées. C'est probablement à ce titre que ses populations méritent d'être sauvées.

\section{Comment et pourquoi sauver la peau de l'ours en Pyrénées françaises?}

\section{VALORISATION TOURISTIQUE DE L'OURS : DÉRIVES ET POSSIBILITÉS}

La pérennité du programme pose parfois question. Le fait d'intégrer pleinement le retour du plantigrade dans une logique touristique pourrait-il assurer sa pérennité? Il ne faut pas sous-

5. En Slovénie, une trentaine d'ours sont victimes annuellement d'accidents de la route. Dans les Pyrénées occidentales, l'ourse Juliette est morte en tombant dans un ravin au début des années 1980. 
estimer l'impact de l'animal à ce niveau. Certains le démentent et accusent l'ours de représenter une menace pour l'activité touristique. Le danger potentiel qu'il induirait serait un frein à la bonne santé de ce secteur. Pourtant, la réalité dément ces assertions à bien des égards. Pour preuve en est la large demande des badauds, randonneurs, estivants en tout genre qui souhaitent partir sur la trace de l'animal. Les syndicats d'initiative, offices de tourisme sont très souvent et de plus en plus interrogés sur les ours par les vacanciers qui souhaiteraient savoir où il est possible de les observer.

L'animal n'est cependant pas facilement visible. Il est rare de l'apercevoir et l'espèce craint le dérangement. Elle attire cependant. En résulte une importante utilisation de son effigie au travers de cartes postales, guides touristiques, toponymie récente... Son succès est incontestable auprès des adeptes du milieu pyrénéen.

Réfléchir aux solutions qui pourraient permettre à l'ours de se maintenir durablement dans le massif pourrait aller dans le sens d'une perspective d'utilisation touristique plus importante encore. Le risque de «touristisation » n'est pas loin : la marchandisation pure et simple de l'animal perdrait tout intérêt environnemental et
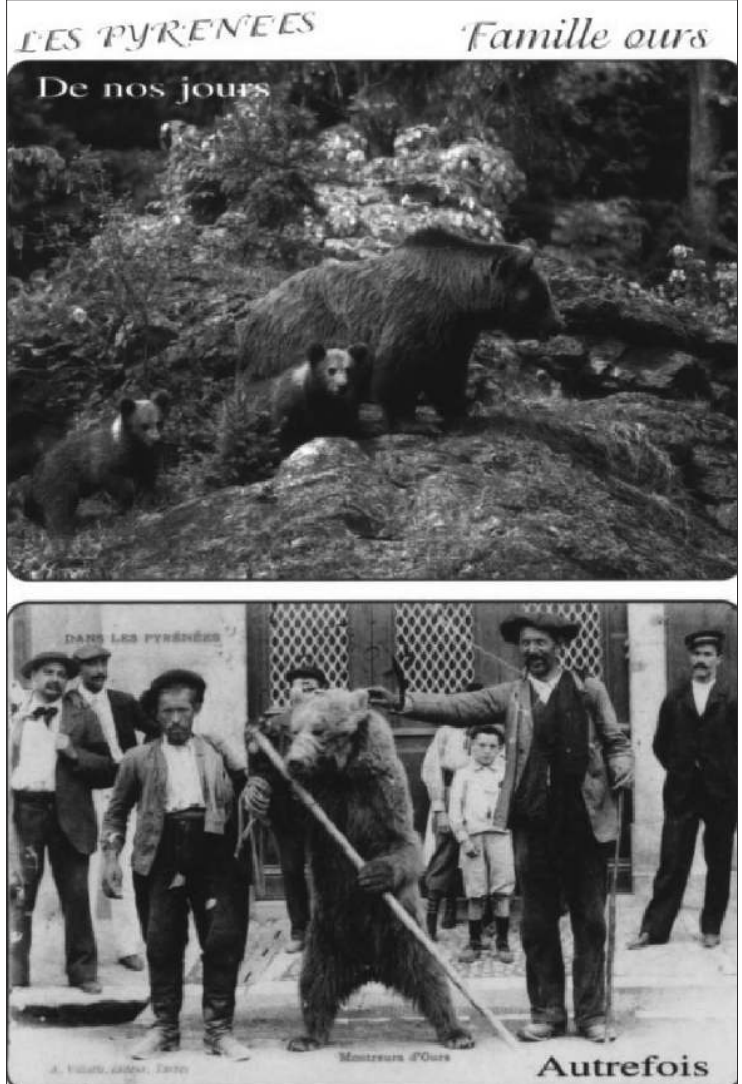

Photo 2 : Carte postale présentant les évolutions du statut de l'ours dans les Pyrénées (cartes postales)

The evolution of Bear status in the Pyrenees discréditerait le but écologique de cette politique. Des dérives méritent d'être relevées et posent question en terme d'éthique. L'ours commence aussi à être victime de son succès. À titre d'exemple, on peut citer l'essor d'établissements touristiques appelés "Maison de l'Ours ». Si l'aspect pédagogique est mis en avant, la marchandisation est là. Celle de Saint-Lary (Hautes-Pyrénées), notamment, fait l'objet d'une controverse. Deux ours y sont captifs. Sauvés d'un cirque, ils vivent maintenant dans un enclos exigu et bétonné.

Certes, ces animaux ne sont plus autonomes, mais un environnement plus adapté à l'animal apporterait plus de cohérence à l'établissement et aux buts affichés : sensibilisation, informations autour de différents outils pédagogiques. La situation a ému beaucoup de gens qui se sont mobilisés pour des conditions de vie plus décentes pour les deux ursidés.

L'écotourisme, plus discret et en plein essor constituerait une solution à développer. De ce point de vue, la présence de l'ours est légitime et induit déjà des retombées positives pour les activités locales (Marsat, 2004; Benhammou, 2005). Grâce au travail de l'ADET-Pays de l'ours, une valorisation touristique a été mise en place. Des chartes de qualité et le label «Pays de l'ours » ont été adoptés par des aubergistes, restaurateurs et professionnels du tourisme des Pyrénées centrales. Les accompagnateurs en montagne de Haute-Garonne, d'Ariège et des Pyrénées-Orientales mettent en avant l'animal dans des sorties thématiques. Ils n’hésitent pas à se positionner en tant que protecteurs à part entière de l'ours, comme le fait par exemple Antoine Glory, président des accompagnateurs des Pyrénées-Orientales : «Souvent nous avons des discussions âpres avec des représentants de la 


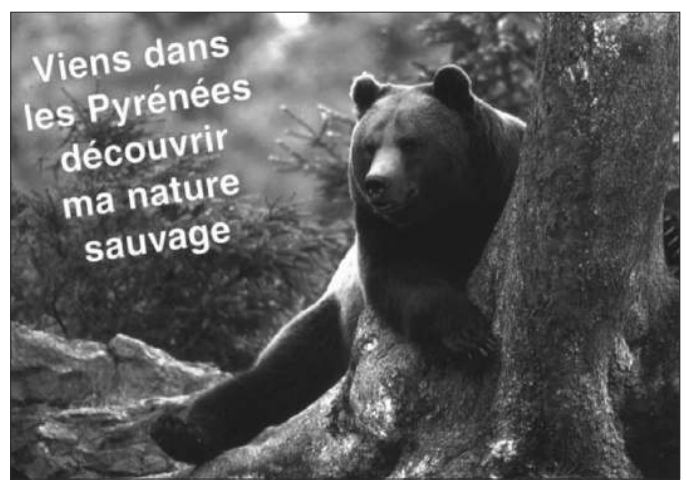

Photo 3 : L’ours, élément touristique attractif (carte postale) The Bear, an attractive and tourist element

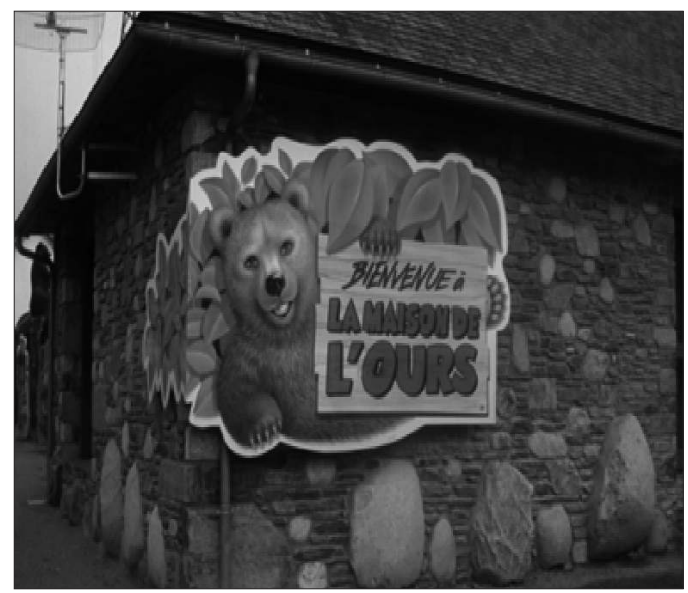

Photo 4 : La maison de l'ours à Saint-Lary (Hautes-Pyrénées) (Source : M. Coquet, décembre 2006)

Pyrénées)

The House of the Bear in Saint Lary (Hautes-

profession agricole qui disent qu'il s'agit d'une idée de technocrates ou d'écolos de Paris. Mais nous, on dit que ce ne sont pas les seuls, nous qui vivons au pays, nous voulons entrer dans le débat et faire entendre notre voix favorable à la protection de ces espèces » (in Benhammou et Salvador, 2003). Des garanties de non dérangement de l'espèce ont en outre été apportées.

De point de vue économique, les raisonnements allant dans le sens de sa sauvegarde sont nombreux. Près de 150 emplois directs, temps plein ou saisonniers, résultent du programme de restauration de l'ours dans les Pyrénées. Si on se penche notamment sur le pastoralisme, il est possible de dégager des interrelations systémiques positives initiées par le retour de l'ours.

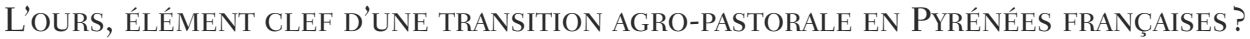

L'hypothèse du retour de l'ours comme atout pour le pastoralisme peut paraître provocante. Elle n'est pas néanmoins dénuée de sens si elle est prise dans le cadre d'une mutation globale du système. Le modèle agro-pastoral, dont la crise s'est amorcée depuis les années 1950-1960, arrive à son point de rupture et doit maintenant réussir sa mutation. Sur les près de 2 millions d'euros annuels consacrés par le ministère de l'Écologie au programme ours, $60 \%$ du budget concernent directement le pastoralisme. À ceux qui crient au « gaspi », rappelons que cela est modique par rapport aux énormes budgets du ministère de l'Agriculture (de l'ordre de 30 milliards dont 11 de subventions européennes à distribuer).

L’ours peut représenter l'élément central entraînant différentes conséquences positives pour l'espace pyrénéen. D’abord, il permet de débloquer différentes aides telles que le gardiennage permanent (par un berger) et de se munir de chiens de protection (Patous). Ainsi, la mortalité due à de mauvaises conditions sanitaires diminue dans le troupeau, il est mieux soigné et il est protégé contre les prédations de tous types. Les professionnels de l'élevage ayant adopté ces mesures constatent une diminution des pertes de bétail par rapport à la période précédant le retour du plantigrade en Pyrénées centrales ${ }^{6}$. Une boutade paradoxale pourrait même résumer la situation : l'ours sauve des brebis! La profession de berger est clairement encouragée dans le massif. Avec une centaine d'emplois de bergers financés par le ministère de l'Écologie au titre du plan ours,

6. Sur un cheptel de 600000 ovins fréquentant le massif pyrénéen, les estimations des pertes annuelles toutes causes confondues vont de 10000 à 28000 bêtes par an (Lions, 1998; Reynes, 2005). 
c'est toute la masse salariale du pastoralisme de montagne qui augmente. Dans un milieu habitué au paiement au noir de salaires modiques, les réticences des éleveurs employeurs sont éclairées d'un jour nouveau face à ce qui est rarement présenté comme une nouvelle ingérence administrative. De même, le Patou, grand chien blanc emblématique de ces montagnes, amorce un retour, comme élément de patrimoine réintroduit. Ce chien ancestral, dont la seule mission est de protéger le troupeau contre toute agression, a connu une rénovation de son utilisation, notamment en Amérique du Nord et dans le Massif central. Symbole d'archaïsme pour les uns habitués à la mécanisation et à la déshumanisation du travail agricole, il peut apparaître comme l'emblème d'une modernité pastorale à créer pour sauvegarder l'agriculture de montagne. En effet, il n'est pas éthiquement tenable d'attendre d'un berger qu'il s'enroule dans une peau de mouton pour veiller sur ses bêtes par tous les temps, comme le font encore des bergers de Roumanie ou d'Albanie. Les dispositifs de gardiennage proposés par le programme ours, ajoutés à ceux préexistants, cherchent à accompagner les adaptations de la profession.

Cela peut s'articuler avec la création de nouveaux labels liés au retour de l'ours, comme le fromage Pé descaous en Béarn et la viande d'agneau des Broutard du Pays de l'ours en Pyrénées centrales (fig. 4). Ces produits se fondent sur des critères qualitatifs. Ainsi, les éleveurs, en se fédérant et en profitant de la contribution d'associations (Fonds d'intervention éco-pastoral, ADET-Pays de l'ours) valorisent leur production et sa distribution. D'une logique de quantité, encore trop présente en montagne, l'aspiration à des produits davantage qualitatifs grandit. Le produit est vendu à un prix permettant une meilleure rémunération de l'agriculture pastorale, qui peut favoriser à terme l'indépendance vis-à-vis des aides étatiques. En lien avec ces processus, l'écotourisme, sensible à la préservation et à la restauration de l'environnement, peut représenter un facteur économique dynamisant.

\section{Conclusion}

En 2008, le nombre d'ours est d'un peu plus d'une vingtaine dans les Pyrénées. Les attaques du prédateur ou les mésaventures des plantigrades occupent trop souvent le devant de la scène, ce qui facilite les schémas d'analyse caricaturaux. Pourtant, les éléments d'une dualité sociale et environnementale de l'agriculture de montagne sont révélés par cette question de l'ours.

Si l'animal n'est pas responsable de la fin du pastoralisme, il représente néanmoins une contrainte réelle. Mais ses opposants se trompent d'ennemis en accusant l'ours d'être responsable de tous les maux de l'agriculture de montagne. Celle-ci est en passe de disparaître, un productivisme paradoxalement lié à l'extensivité ayant eu systématiquement tendance à faire disparaître les exploitations agricoles. Contrairement aux messages simplistes véhiculés par les opposants à l'espèce, la réintroduction de l'ours ouvre des pistes de réflexions pertinentes pour l'avenir de cette région. Le retour du prédateur oblige en effet à un retour de l'homme en montagne. Or, qui dit hausse de la présence de berger, dit véritable entretien des paysages tant mis en avant. Le fait que ce soit d'abord un ministère de l'Écologie désargenté qui vienne à la rescousse du pastoralisme ne doit pas faire oublier sa dimension agricole. Cette espèce emblématique qu'est l'ours semble incarner la manière dont toute problématique environnementale devrait être traitée : une articulation systémique entre tous les acteurs concernés, n’omettant pas la préservation des caractéristiques culturelles et surtout écologiques des territoires. Pour cela, il ne suffit pas de se contenter des discours de surface de quelques leaders médiatisés. Un travail d'analyse de fond est indispensable. 


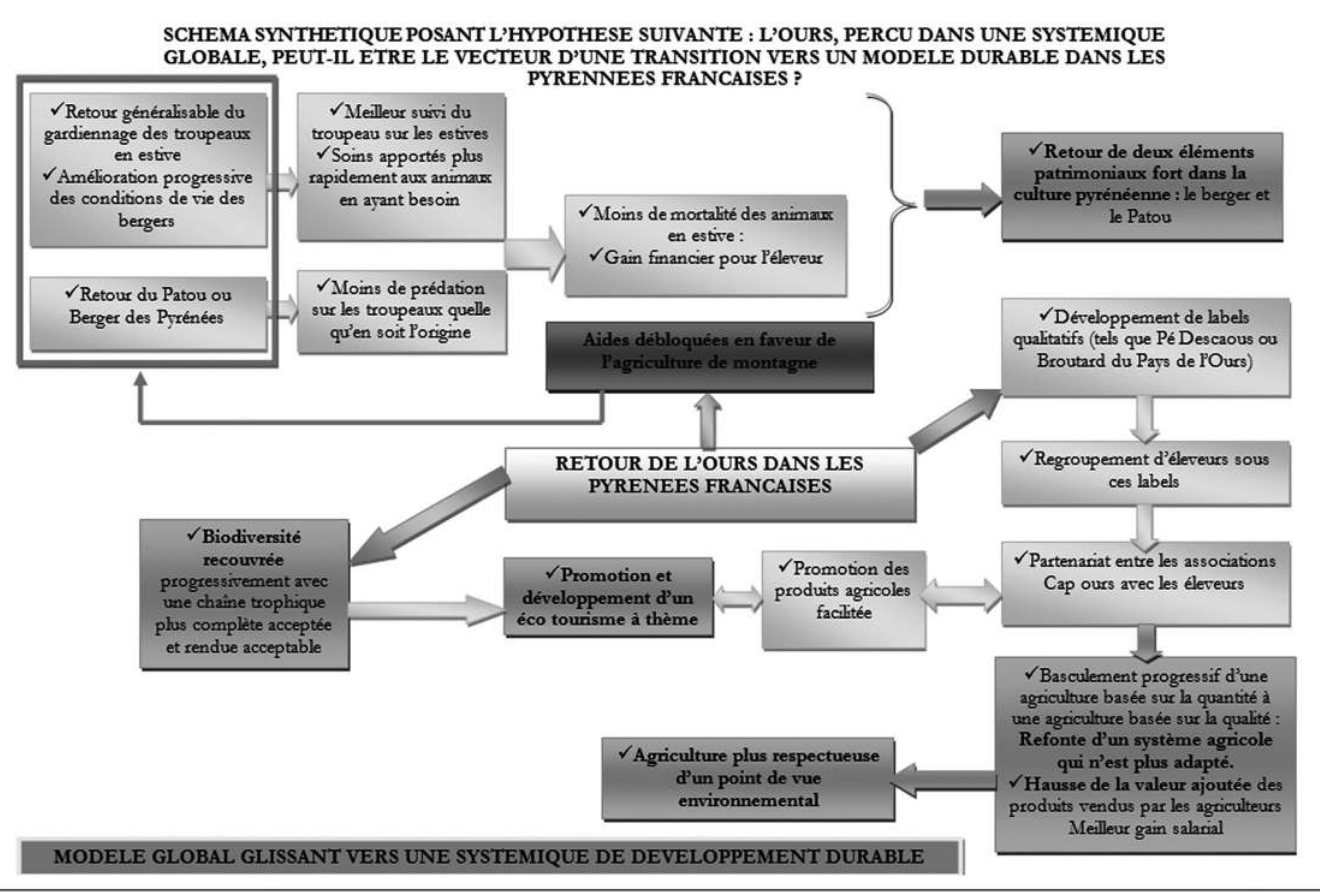

\section{Point de départ des hypothèses}

Aides diverses (financières, matérielles ...) apportées à Pagriculture de montagne

Ce que cela engendre ou pourrait engendrer

Eléments conduisant à une mutation qualitative terntorialisée

Concerne

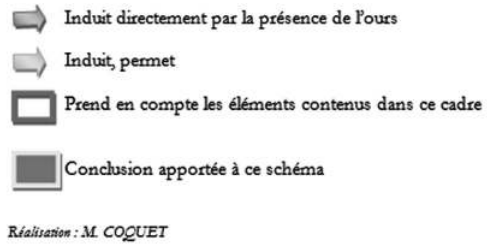

$\Rightarrow$ Induit directement par la présence de Pours

Figure 4 : L’ours, vecteur d'une transition vers un modèle durable dans les Pyrénées françaises? The Bear, a way to a sustainable model of developement?

\section{Bibliographie}

Bailly G., ForTASsin F., 2008. - Revenons à nos moutons : un impératif pour nos territoires et notre pays, Rapport d'information du Sénat, Commission des Affaires économiques, disponible sur : [http://www.senat. fr/rap/r07-168/r07-168_mono.html\#toc397].

Bellefon (De) (R.), Benhammou (F.), 2004. - « Les limites d'un discours environnementaliste : la Confédération paysanne et les prédateurs », dans Benhammou (F.), Baillon (J.), Senotier (J.-L.) (dir.), « La cohabitation hommes/grands prédateurs en France (loup et ours) », Recherches Naturalistes en région Centre, $\mathrm{n}^{\circ} 14$, p. 179-188.

Benhammou (F.), 2001. - Rôles et dynamiques des porte-parole dans les débats sur l'ours dans les Pyrénées, analyse stratégique et géopolitique d'un problème d'environnement, mémoire de DEA Aménagement-Développement-Environnement, IRD, Université d'Orléans-ENGREF de Paris, 131 p.

—, 2003. - « Les grands prédateurs contre l'environnement? Faux enjeux pastoraux et débat sur l'aménagement des territoires de montagne », Courrier de l'Environnement de l'INRA, février 2003, n 48, p. 5-12. 
—, 2005, - « Vendre la peau de l'ours avant de l'avoir sauvé? Une géopolitique locale de la conservation d'une espèce animale emblématique », dans Benhammou (F.), Bobbe (S.), Camara (J.-J.), Reynes (A.) (dir.), L'ours des Pyrénées, les 4 vérités, Toulouse, Privat, p. 77-120.

—, 2006, - « Territoires des animaux, territoires des hommes : aspects et enjeux du retour des grands prédateurs en France », Géoconfluences, Brèves n 3, 2006 : [http://geoconfluences.ens-lsh.fr/doc/breves/2006/3. $h t m]$.

—, 2007. - Crier au loup pour avoir la peau de l'ours, Une géopolitique locale de l'environnement à travers la gestion et la conservation des grands prédateurs en France, Thèse de $3^{\mathrm{e}}$ cycle, Agro Paris Tech'-ENGREF, École Nationale du Génie Rural des Eaux des Forêts, 649 p.

Benhammou (F.), Mermet (L.), 2003. - « Stratégie et géopolitique de l'opposition à la conservation de la nature : le cas de l'ours des Pyrénées », Natures, Sciences, Sociétés, nº 11, p. 381-393.

Benhammou (F.), Salvador (O.), 2003. - « Le loup (Canis lupus) dans les Pyrénées, 1998-2003 : cas d'école pour anticiper le retour des prédateurs sauvages dans les territoires ruraux marginaux, Sud-Ouest Européen ", Revue de Géographie des Pyrénées et du Sud-Ouest, n 16, p. 85-93.

Blanc (N.), Cohen (M.), 2001. - "L'animal, une figure de la géographie contemporaine », Espaces et Sociétés, n 110-111, p. 25-40.

BobBe (S.), 1998. - Du folklore à la science. Analyse anthropologique des représentations de l'ours et du loup dans l'imaginaire occidental, Thèse de $3^{\mathrm{e}}$ cycle, EHESS, Paris, $420 \mathrm{p}$.

Camarra (J.-J.), 2005. - « Biologie, écologie de l'ours brun », dans Benhammou (F.), Bobbe (S.), Camara (J.-J.), Reynes (A.), L'ours des Pyrénées, les 4 vérités, Toulouse, Privat, p. 15-36.

Charlier (B.), 1988. - « L'animal et les enjeux historiques et contemporains de l'aménagement de l'espace montagnard dans les Pyrénées occidentales », Sud-Ouest européen, n 3, p. 47-54.

Coquet (M.), 2007. - L'ours brun et les sociétés rurales montagnardes des Pyrénées françaises, des rapports complexes, mémoire de Master 2 Sociétés et Régulations, Université d'Angers, 155 p.

Dendaletche (C.), 1993. - La cause de l'ours, Paris, Sang de la Terre, 228 p.

Etchelecou (A.), 1995. - « Ours bruns des Pyrénées occidentales. Éléments d'analyse démographique », IX Conférence Internationale, Connaissance et gestion des populations d'ours, Gestion et restauration de petites populations et populations reliques, 19-22 octobre 1992, Paris-Grenoble, ministère de l'Environnement-Muséum d'histoire naturelle de Grenoble, p. 97-107.

Lamazou (E.), 1988. - L'ours et les brebis, Paris, Payot, 203 p.

Lions (S.), 1998. - Pastoralismes Pyrénées, Rapport d'étude, AGROMIP-DATAR/Commissariat à l'aménagement des Pyrénées.

Marliave de (O.), 2000. - Histoire de l'ours dans les Pyrénées, de la préhistoire à la réintroduction, Bordeaux, Sud-Ouest, $254 \mathrm{p}$.

Marsat (J.-B.), 2004. - « Le tourisme et la gestion de l'ours », dans Benhammou (F.), Baillon (J.), SenoTIER (J.-L.) (dir.), «La cohabitation hommes/grands prédateurs en France (loup et ours)», Recherches naturalistes en région Centre, $\mathrm{n}^{\circ}$ 14, p. 133-138.

Mermet (L.), 2001. - «L'Institution patrimoniale du Haut Béarn : gestion intégrée de l'Environnement ou réaction anti-environnementale? ", Responsabilité Environnement, n 21, p. 9-21.

Mermet (L.), 2002. - «L'Homme ou la vie sauvage? La société locale ou la bureaucratie centrale? : faux dilemmes et vrais rapports de force », Responsabilité Environnement, n 28, p. 13-21.

Mermet (L.), Benhammou (F.), 2005. - « Prolonger l'inaction environnementale dans un monde familier : la fabrication stratégique de l'incertitude sur les ours du Béarn », Écologie et politique, n 31, p. 121-136.

Mermet (L.), 2006 [1998]. - L'analyse stratégique de la gestion environnementale - illustrée par les tribulations d'un noyau relictuel de population d'ours brun dans les Pyrénées occidentales françaises, Paris, ENGREFRGTE, 484 p.

Pejetnov (V.), 1998. - Avec les ours, Arles, Actes Sud, 231 p.

Paroix (J.), Faucompret (J.-M.), 1999. - Berger dans les nuages, Pau, Éditions de Faucompret, 112 p.

Pinet (C.), 2006. - « Loups de France et du Canada : essai sur les représentations, usages et gestion d'un prédateur en milieu montagnard », ESO, n²5, p. 75-83. 
QuenetTe (P.-Y.), 2000. - « Bilan scientifique et technique de la réintroduction de l'ours en Pyrénées centrales », synthèse des données 1996-2000, Équipe de suivi DIREN Life, MATE, DIREN Midi-Pyrénées, $155 \mathrm{p}$.

Reynes (A.), 2005. - « De la chasse à la réintroduction : entre espoir et histoire », dans Benhammou (F.), Bobbe (S.), Camara (J.-J.), Reynes (A.), L'ours des Pyrénées, les 4 vérités, Toulouse, Privat, p. 39-78.

Cet article a été reçu le 12 septembre 2008 et définitivement accepté le 23 octobre 2008. 